\title{
Efecto de diferentes combinaciones de temperatura y salinidad sobre el consumo específico de oxígeno en el camarón blanco Litopenaeus vannamei
}

Effect of different combinations of temperature and salinity on specific oxygen consumption in the white shrimp Litopenaeus vannamei

\section{Wenceslao Valenzuela-Quiñónez ${ }^{1}$, Gerardo Rodríguez-Quiroz ${ }^{1}$, Jesús T. Ponce-Palafox ${ }^{2}$ y Héctor M. Esparza-Leal ${ }^{1}$}

\begin{abstract}
${ }^{1}$ Centro Interdisciplinario de Investigación para el Desarrollo Integral Regional-IPN, Unidad Sinaloa, Boulevard Juan de Dios Bátiz Paredes \#250, Guasave, Sinaloa 81101, México. hesparza@ipn.mx

${ }^{2}$ CENITT, Posgrado CBAP, Escuela Nacional de Ingeniería Pesquera, Universidad Autónoma de Nayarit, Ciudad de la Cultura Amado Nervo, Tepic, Nayarit 62155, México
\end{abstract}

\begin{abstract}
The combined effect of different temperatures $\left(20,25\right.$ and $\left.30^{\circ} \mathrm{C}\right)$ and salinities $(15,25,35$ and 45 psu) on the specific oxygen consumption (CEO) in Litopenaeus vannamei was determined. For this, CEO of 248 organisms of different body sizes ( 0.31 to $15.85 \mathrm{~g})$ was measured. The CEO decreased when shrimp weight increased in all treatments without presenting a definite pattern when the salinity increased; even though all the combinations of temperature and salinity did not statistically show slopes significantly different in the regression models for CEO and organism weight relationships. The slope values suggest that at $20^{\circ} \mathrm{C}$ there is high energy expenditure at $15 \mathrm{psu}$, and at $25^{\circ} \mathrm{C}$ the highest energy consumption occurs at $45 \mathrm{psu}$. Whereas at $30^{\circ} \mathrm{C}$, the lowest energy consumption occurred combined with the lowest salinity (15 psu). This may indicate that the isosmotic condition (point of lowest energy expenditure) tends to change with temperature, which may imply that the shrimp can be cultivated at high temperature $\left(30^{\circ} \mathrm{C}\right)$ and at low salinities (15 psu) without increasing energy expenditure.
\end{abstract}

Key words: Respiratory rate, energy expenditure, abiotic variables, Mexico

Resumen.- Se analizó el efecto combinado de diferentes temperaturas $\left(20,25\right.$ y $\left.30^{\circ} \mathrm{C}\right)$ y salinidades $(15,25,35$ y 45 ups) sobre el consumo específico de oxígeno (CEO) en Litopenaeus vannamei. Se midió el CEO en 248 organismos de distintas tamaños corporales (0,31 a 15,85 g). El CEO disminuyó con el aumento de peso corporal de los organismos en todos los tratamientos, sin observarse un patrón general definido de aumento del CEO conforme se incrementó la salinidad. Aunque no hubo diferencias estadísticas significativas en todas la combinaciones de temperatura-salinidad entre las pendientes de regresión que relacionan el CEO con el peso de los organismos, los valores de pendientes en particular sugieren que en $20^{\circ} \mathrm{C}$ se da un mayor gasto energético en la menor salinidad (15 ups) y en $25^{\circ} \mathrm{C}$ en la salinidad más alta (45 ups). Mientras que en $30^{\circ} \mathrm{C}$, el menor gasto energético se presentó en la menor salinidad (15 ups). Lo cual puede indicar que la condición isosmótica (punto de menor gasto energético) tiende a modificarse con la temperatura, favoreciendo con ello que los camarones se puedan cultivar en alta temperatura $\left(30^{\circ} \mathrm{C}\right)$ y baja salinidad (15 ups) sin que se presente un mayor gasto energético.

Palabras clave: Tasa respiratoria, gasto energético, variables abióticas, México

\section{INTRODUCCIÓN}

La oxigenación del agua es un punto crítico para los acuícultores, dado que en pocos minutos una disminución de la concentración de oxígeno disuelto en el agua hasta el nivel mínimo tolerado por los camarones puede provocar su muerte (Boyd \& Watten 1989, Re \& Díaz 2011). El oxígeno es esencial para el metabolismo de los organismos debido a que participa en diferentes procesos oxídativos de liberación de energía y puede afectar su alimentación, crecimiento y reproducción (Salvato et al. 2001).
El consumo de oxígeno es una respuesta fisiológica que puede correlacionarse con las variaciones de los factores ambientales, debido a que la tasa respiratoria está relacionada con el trabajo metabólico y el flujo de energía que los organismos canalizan hacía los mecanismos del control homeostático (Salvato et al. 2001). Dentro de las variables ambientales, la temperatura y salinidad son las que más influyen sobre los camarones, ya que la temperatura afecta directamente a todos los 
procesos biológicos, mientras que la salinidad afecta la demanda osmorregulatoria (Yan et al. 2007, Valdez et al. 2008). Ambas variables afectan el contenido de oxígeno del medio y tanto el efecto de una sola variable como la interacción de ambas, salinidad y temperatura, puede ser complejo, ya que una variable puede actuar como factor modulador del efecto de la otra (Vernberg 1983).

Se han diseñado sistemas electrónicos sofisticados que ayudan a controlar el nivel de concentración de oxígeno en los sistemas de cultivo (Summerfelt et al.2000). Sin embargo, se requiere ampliar el conocimiento de las tasas respiratorias de los organismos bajo diferentes condiciones ambientales, con el fin de mantener niveles óptimos de oxígeno en función de la máxima producción al mínimo costo.

La evaluación del consumo de oxígeno en camarones expuestos a diferente temperatura y salinidad es importante, debido a que a nivel de cultivo estas dos variables abióticas son las que presentan mayor variación $\mathrm{y}$, el consumo de oxígeno es un factor que indica la cantidad de energía consumida (Rosas et al. 1997, Re \& Díaz 2011). Por lo cual, para optimizar la producción en los sistemas de cultivo ésta información es útil para estimar los requerimientos iníciales del flujo de agua en función de la densidad de organismos.

Varios investigadores han estudiado el efecto de la temperatura y salinidad sobre el consumo de oxígeno en los camarones peneidos (Martínez-Palacios et al. 1996, Rosas et al. 1997, Re et al. 2004, Spanopoulos-Hernández et al. 2005, Li et al. 2007, Bett \& Vinatea 2009) y se ha determinado que la respuesta metabólica varía dependiendo la etapa del ciclo de vida y variaciones fisiológicas propias de la especie.

En Penaeus brasiliensis, Robaina (1983) y Scelzo \& Zuñiga (1987) evaluaron la respiración en función del peso, salinidad, temperatura $\mathrm{y}$, determinaron que el consumo de oxígeno se relacionó con el peso de los organismos y se incrementó a medida que la temperatura y salinidad aumentaron. Lo mismo fue documentado en trabajos realizados con $P$. indicus (Kutty et al. 1971), $P$. monodon (Liao \& Murai 1986, Kurmaly et al. 1989), $P$. japonicus (Chen \& Lai 1993) y Litopenaeus stylirostris (Spanopoulos-Hernández et al. 2005).

Existen pocos estudios sobre la fisiología respiratoria de Litopenaeus vannamei, dentro de los que se pueden mencionar el efecto de la temperatura $\left(20,24,28\right.$ y $\left.32^{\circ} \mathrm{C}\right)$ y salinidad $(25,30,35,40$ y 45 ups) sobre el consumo de oxígeno de postlarvas (Villarreal \& Rivera 1992) y, el efecto de la temperatura $\left(20,25\right.$ y $30^{\circ} \mathrm{C}$ a 35 ups) sobre el consumo de oxígeno de juveniles y adultos (Martínez-Palacios et al. 1996). En estos estudios se determinó que la temperatura tuvo un efecto directo sobre el consumo de oxígeno de juveniles y adultos, mientras que en las postlarvas la salinidad no afectó dicho consumo. En el presente estudio, el objetivo fue determinar el efecto de diferentes combinaciones de temperatura $\left(20,25\right.$ y $\left.30^{\circ} \mathrm{C}\right)$ y salinidad $(15,25,35$ y 45 ups) sobre el consumo específico de oxígeno (CEO) en juveniles de camarón blanco $L$. vannamei, para aportar información que permita estimar los requerimientos de flujo de agua en función de las tasas respiratorias bajo diferentes condiciones de temperatura y salinidad en la principal etapa de desarrollo del camarón $(0,31$ a 15,85 g) de los cultivos comerciales del noroeste de México.

\section{Materiales y MÉtodos}

Se experimentó con 12 tratamientos, resultado de la combinación de tres temperaturas $\left(20,25\right.$ y $\left.30^{\circ} \mathrm{C}\right)$ y cuatro salinidades $(15,25,35$ y 45 ups), cuyos valores están dentro de los intervalos en los que fluctúan estas variables en las granjas camaronícolas del noroeste de México.

Se construyó un respirómetro de flujo cerrado de acuerdo al modelo de Ross \& Ross (1983). Para su implementación se utilizó un acuario de plástico con capacidad de $30 \mathrm{~L}$ en el que se podían manejar cuatro cámaras de prueba $(\mathrm{CP})$. Se utilizaron tres juegos de $\mathrm{CP}$ (cuatro cámaras por juego) de 70, 300 y $600 \mathrm{~mL}$ de capacidad, que se adecuaban para los organismos experimentales pequeños, medianos y grandes, respectivamente. El volumen total de los sistemas cerrados fluctúo en un intervalo de $370-970 \mathrm{~mL}$ y la tasa de flujo en un intervalo de $37-146 \mathrm{~mL} \mathrm{~min}^{-1}$. El flujo de agua en el sistema se mantuvo mediante 4 bombas centrífugas con implemento magnético (una bomba para cada sistema de recirculación; Eheim ${ }^{\circledR}, 115$ volts, Lancaster, PA, USA). Dentro del acuario se instaló un difusor de aire para mantener homogénea la concentración de oxígeno y a la entrada de agua de cada CP se le puso un filtro de malla de $120 \mu \mathrm{m}$ para impedir el paso de burbujas de aire. La cámara de medición de oxígeno (CMO) consistió de un recipiente de vidrio que se mantuvo cerrado, dentro del cual se colocó un agitador magnético que se activó para mantener homogénea el agua de la cámara y a su vez tenía insertado el electrodo de un oxímetro (YSI 55 oxygen meter, Yellow Springs Instrument Co., Inc., Yellow Springs, OH, USA). La comunicación entre las CP y la CMO se dio a través de un sistema de 
mangueras de hule $($ diámetro $=1 / 4 ")$ y válvulas de dos vías. Durante la operación del sistema, el agua se bombeó de la CP hacia el acuario de plástico o hacia la CMO. Cuando se realizó la medición del consumo de oxígeno se cerraba el sistema por medio de las válvulas de tal manera que se estableció un circuito cerrado para realizar la medición. Diariamente, se revisó la membrana del sensor del oxímetro y se calibró antes de llevar a cabo la medición. La calibración del oxímetro se realizó diariamente de acuerdo a las especificaciones del equipo y quincenalmente con el método Winkler (Grasshoff 1976). La salida analógica del oxímetro se mantuvo conectada a una computadora para captar la información en el programa Procomm versión 2.4.3 (PCWorld Communications, San Francisco, CA, USA), que producía un archivo de datos que se exportaba a una hoja de cálculo para su análisis posterior. La temperatura del agua del acuario se midió diariamente con un termómetro de mercurio (Branan Company ${ }^{\circledR}$, Cumbria, UK), al igual que la salinidad con un refractómetro (American Optical Company $^{\circledR}$, Southbridge, MA, USA).

Se utilizó agua marina bombeada directamente de la orilla del mar desde una galería filtrante ubicada en la playa y agua marina preparada con salmuera para alcanzar la mayor salinidad del estudio. Para evitar la demanda biológica de oxígeno (DBO) por bacterias en el sistema de recirculación durante el trabajo experimental, antes de utilizar el agua se filtró con UV y posteriormente se le aplicó cloro al 5\% (3 $\left.\mathrm{mL} \mathrm{L}^{-1}\right)$ dejándola reposar por $24 \mathrm{~h}$, para posteriormente neutralizar el cloro con tiosulfato de sodio $\left(150 \mathrm{mg} \mathrm{L}^{-1}\right)$, dándole un reposo final por $\approx 24 \mathrm{~h}$ (Hemerick 1973). El respirómetro completo (cámaras, mangueras y recipientes) se lavó semanalmente con cloro al $10 \%$. Para tomar en cuenta el posible consumo de oxígeno por los microorganismos presentes en el agua, durante cada uno de los bioensayos se midió el consumo de oxígeno en una cámara de prueba sin organismo experimental. Dicho consumo se le restó al consumo del organismo experimental en turno.

Se obtuvieron 2 lotes de camarón Litopenaeus vannamei, uno de postlarvas de un laboratorio comercial y otro de una granja camaronícola que había sido abastecida de postlarvas por el mismo laboratorio, con el fin de mantener una diversidad de tamaños corporales, de donde se utilizaron 248 juveniles para todos los tratamientos, que fluctuaron entre 0,31 y $15,85 \mathrm{~g}$ de peso húmedo.

Previo a la experimentación, los organismos se mantuvieron en un laboratorio húmedo en un sistema de recirculación de agua acondicionado con un filtro biológico durante 15 días. Dicho sistema constó de 4 tinas de $600 \mathrm{~L}$ de capacidad cada una. Cada tina se mantuvo con aireación constante y a los organismos se les suministró alimento ad libitum dos veces al día (alimento comercial para camarón marca PIASA: $35 \%$ de proteína, $8 \%$ de grasa, $3 \%$ de fibra, $12 \%$ de humedad, $48 \%$ de ELN). El laboratorio se mantuvo con un fotoperiodo de 12L:12O (la fotofase comenzó a las 06:30 a.m.).

Las condiciones del laboratorio húmedo fueron controladas de acuerdo al experimento en turno. Se manipuló la temperatura con tres calentadores eléctricos y dos aires acondicionados de 18,000 BTU cada uno, para mantenerla en un intervalo de 17 a $33^{\circ} \mathrm{C}$ y en el agua de las tinas y respirómetro entre 19 y $32^{\circ} \mathrm{C}$. Según el experimento en turno, los organismos se aclimataron a cada una de las temperaturas $\left(20,25\right.$ ó $\left.30^{\circ} \mathrm{C}\right)$ y salinidades $(15,25,35$ ó 45 ups) por un mínimo de 1 semana antes de cada experimento, con el fin de que no influyera la aclimatación en el consumo de oxígeno de los organismos (Martínez-Palacios et al. 1996). La aclimatación de los organismos a las diferentes temperaturas se llevó a cabo manipulando gradualmente la temperatura del laboratorio $\left(\approx 5^{\circ} \mathrm{C} 24 \mathrm{~h}^{-1}\right)$, de tal manera que el agua de las tinas en las que se mantenían los organismos estuviera en la temperatura correspondiente al experimento en turno. De igual manera, los organismos se aclimataron gradualmente a la salinidad de 15, 25, 35 ó 45 ups $\left(\approx 1\right.$ ups h$^{-1}$; EsparzaLeal et al. 2010) según el experimento llevado a cabo. A la salinidad de 45 ups, se aclimató utilizando agua previamente preparada con salmuera $(\approx 55 \mathrm{ups})$, con la misma razón de cambio utilizada en el resto de las salinidades.

A todos los camarones utilizados en la experimentación se les suspendió el alimento por $48 \mathrm{~h}$ antes de depositarlos en las cámaras de prueba, para evitar alteración en el consumo de oxígeno por el metabolismo de la digestión (Scelzo \& Zuñiga 1987). Además, con el fin de evitar el consumo de oxígeno por el efecto de variables diferentes a la temperatura y salinidad, se seleccionó a los organismos experimentales utilizando tres criterios: 1) utilización de los individuos más resistentes, los cuales fueron seleccionados luego de someterlos a cambios bruscos de salinidad, 2) revisión general de los organismos, para descartar los que presentaban necrosis en la cutícula y 3) selección sólo de organismos que estaban en la etapa de intermuda. La etapa de muda de los camarones se identificó con la técnica reportada por Robertson et al. (1987). 
Antes de iniciar cada experimento, cada uno de los camarones fueron pesados en una balanza granataria digital (Ohaus, Pine Brook, NJ, USA), previo a la eliminación del exceso de agua con papel secante, posteriormente se introdujeron a las $\mathrm{CP}$ por un período de 4 a $5 \mathrm{~h}$, tiempo que se consideró suficiente para su recuperación del estrés por manejo (Martínez-Palacios et al. 1996). Una vez estable y a saturación el oxígeno en las $\mathrm{CP}$, se cerró el circuito del respirómetro y se realizaron las mediciones del consumo de oxígeno por un lapso de 7 a $10 \mathrm{~min}$, en total obscuridad. Transcurrido este tiempo se abrió el circuito y posteriormente se retiraban los organismos.

Para cada combinación experimental de temperatura (20-25, 20-30 y $\left.25-30^{\circ} \mathrm{C}\right)$, se obtuvo el coeficiente termal para la tasa metabólica del camarón $\left(\mathrm{Q}_{10}\right)$ en dos grupos extremo (pesos de 1 y $15 \mathrm{~g}$ ), con el fin de determinar la sensibilidad de los organismos a la variación de temperatura. Para ello se utilizó la ecuación propuesta por Schmidt-Nielsen (1997):

$$
\mathrm{Q}_{10}=\left(\mathrm{R}_{2} / \mathrm{R}_{1}\right)^{10 / \mathrm{T} 2-\mathrm{T} 1}
$$

Donde: $\mathrm{R}_{1}$ y $\mathrm{R}_{2}$ son las tasas metabólicas (CEO) a las temperaturas $\mathrm{T} 1$ y $\mathrm{T} 2$, respectivamente.

Para los cálculos del CEO por organismo $\left(\mathrm{mg} \mathrm{L}^{-1}\right)$, se utilizaron los datos del cambio de concentración de oxígeno entre el tercer y sexto minuto. A partir de esta información se obtuvo el CEO en $\mathrm{mg} \mathrm{O}_{2} \mathrm{~kg}^{-1} \mathrm{~h}^{-1}$ con la siguiente fórmula:

$$
\mathrm{CEO}=\mathrm{A}(\mathrm{B} / 1000) /[(\mathrm{C} / 60) *(\mathrm{D} / 1000)]
$$

Donde: A es el oxígeno consumido en $\mathrm{mg} \mathrm{L}^{-1}$ durante el período de medición; $\mathrm{B}$ es el volumen de agua en $\mathrm{mL}$ de la cámara de prueba; $\mathrm{C}$ es el tiempo de medición en minutos; y $\mathrm{D}$ es el peso (g) húmedo del organismo.

Los CEOs y pesos fueron transformados a logaritmos para homogenizar varianzas, normalizar la distribución de errores y hacer aditivos los efectos de tratamientos (Steel $\&$ Torrie 1980). Con el fin de expresar la dependencia del CEO de los organismos con respecto a su peso, en los diferentes tratamientos estudiados, a cada serie de datos se le aplicó un análisis de regresión simple y se evaluó los residuales de acuerdo a los criterios establecidos por Steel \& Torrie (1980), Sokal \& Rohlf (1981) y Zar (1984). Para determinar diferencias significativas se realizaron análisis de covarianza de las pendientes y ordenadas al origen de los modelos de regresión del CEO de todos los tratamientos (se incluyó un análisis entre las distintas temperaturas sin considerar la salinidad) y, cuando correspondió se identificaron las diferencias significativas entre los grupos utilizando la prueba de Tukey HSD para n desigual (Sokal \& Rohlf 1981).

\section{Resultados}

\section{CEO EN LOS DISTINTOS TRATAMIENTOS}

Los organismos expuestos a $20^{\circ} \mathrm{C}$ en las 4 salinidades, mostraron una tendencia general de disminución del CEO conforme aumentó su peso corporal. Sin embargo, los modelos de regresión simple calculados para el CEO no mostraron diferencias estadísticas significativas en las ordenadas al origen de las cuatro salinidades $\left(\mathrm{F}_{(3,103)}=\right.$ $0,05, P>0,05)$, ni en las pendientes $\left(\mathrm{F}_{(3,100)}=1,50, P>\right.$ $0,05)$, aun cuando estas últimas fluctuaron entre $-0,38$ ( 35 ups) y $-0,72$ (15 ups; Tabla 1$)$.

\begin{tabular}{crcccrr}
\hline \multicolumn{2}{c}{ Tratamiento } & Modelo de regresión & $n$ & $r^{2}$ & $\mathrm{~F}$ & $P$ \\
${ }^{\circ} \mathrm{C}$ & ups & & & & & \\
\hline 20 & 15 & $\log \mathrm{CEO}=5,01-0,72 \log \mathrm{W}$ & 27 & 0,64 & 44,60 & $<0,0001$ \\
“ & 25 & $\log \mathrm{CEO}=4,42-0,56 \log \mathrm{W}$ & 23 & 0,33 & 13,50 & 0,0010 \\
“ & 35 & $\log \mathrm{CEO}=3,75-0,38 \log \mathrm{W}$ & 29 & 0,42 & 19,90 & 0,0001 \\
“ & 45 & $\log \mathrm{CEO}=4,54-0,59 \log \mathrm{W}$ & 29 & 0,53 & 31,00 & $<0,0001$ \\
25 & 15 & $\log \mathrm{CEO}=4,61-0,58 \log \mathrm{W}$ & 23 & 0,31 & 10,10 & 0,0042 \\
“ & 25 & $\log \mathrm{CEO}=4,45-0,55 \log \mathrm{W}$ & 12 & 0,33 & 12,80 & 0,0014 \\
“ & 35 & $\log \mathrm{CEO}=5,39-0,80 \log \mathrm{W}$ & 19 & 0,76 & 71,50 & $<0,0001$ \\
“ & 45 & $\log \mathrm{CEO}=5,62-0,83 \log \mathrm{W}$ & 18 & 0,57 & 31,20 & $<0,0001$ \\
30 & 15 & $\log \mathrm{CEO}=3,23-0,19 \log \mathrm{W}$ & 16 & 0,25 & 7,94 & 0,0095 \\
“ & 25 & $\log \mathrm{CEO}=4,28-0,46 \log \mathrm{W}$ & 22 & 0,26 & 7,88 & 0,0100 \\
“ & 35 & $\log \mathrm{CEO}=4,07-0,37 \log \mathrm{W}$ & 14 & 0,31 & 11,60 & 0,0022 \\
“ & 45 & $\log \mathrm{CEO}=4,11-0,41 \log \mathrm{W}$ & 16 & 0,40 & 15,20 & 0,0007 \\
\hline
\end{tabular}

Tabla 1. Modelos de regresión obtenidos para la relación del consumo específico de oxígeno (CEO) y el peso (W) de juveniles Litopenaeus vannamei en la combinación de tres temperaturas $\left(20,25\right.$ y $\left.30^{\circ} \mathrm{C}\right)$ y cuatro salinidades $(15,25,35$ y 45 ups) / Regression models obtained for the relationship of specific oxygen consumption (CEO) and weight (W) of Litopenaeus vannamei juveniles in using the combination of three temperatures $\left(20,25\right.$ and $\left.30^{\circ} \mathrm{C}\right)$ and four salinities $(15,25$, 35 and $45 \mathrm{psu}$ ) 
A $25^{\circ} \mathrm{C}$, dentro de las mismas salinidades, también los camarones presentaron una disminución del CEO conforme aumentó su peso, sin presentarse diferencias significativas tanto entre las ordenadas al origen de los modelos de regresión $\left(\mathrm{F}_{(3,67)}=2,20, P>0,05\right)$ como entre las pendientes $\left(\mathrm{F}_{(3,64)}=1,00, P>0,05\right)$. De igual manera, cuando los organismos se expusieron en las cuatro salinidades a $30^{\circ} \mathrm{C}$, disminuyó el CEO conforme aumentó el peso de los organismos, aunque en este caso si se presentaron diferencias significativas entre las ordenadas al origen de los modelos de regresión de 15 ups $(P=$ $0,0091)$ y 25 ups $(P=0,0127)$ con 35 ups, pero no entre las pendientes cuyos valores fluctuaron entre -0,19 (15 ups) y $-0,46$ (25 ups; $\mathrm{F}_{(3,60)}=1,50, P>0,05$; Tabla 1$)$.

\section{CEO EN DISTINTAS TEMPERATURAS SIN CONSIDERAR LA SALINIDAD}

El CEO tendió a aumentar según la temperatura del tratamiento, 20,25 a $30^{\circ} \mathrm{C}$ (Fig. 1). En organismos de 5 y 10 g, el CEO aumentó 0,30 veces al pasar de 20 a $25^{\circ} \mathrm{C}$ y 0,48 veces al pasar de 25 a $30^{\circ} \mathrm{C}$. Se presentaron diferencias significativas entre las ordenadas al origen de los modelos de regresión de las tres temperaturas: $20-25^{\circ} \mathrm{C}(P<0,0001)$, $20-30^{\circ} \mathrm{C}(P=0,0105)$ y $25-30^{\circ} \mathrm{C}(P<0,0001)$. Sin embargo, aunque las pendientes de los modelos de regresión fluctuaron entre $-0,34\left(30^{\circ} \mathrm{C}\right)$ y $-0,69\left(25^{\circ} \mathrm{C}\right.$; Tabla 2$)$, no se presentaron diferencias significativas entre éstas: $20-25^{\circ} \mathrm{C}$ $(P<0,0001), 20-30^{\circ} \mathrm{C}(P<0,0001)$ y $25-30^{\circ} \mathrm{C}(P=0,0082)$. Por otro lado, el $\mathrm{Q}_{10}$ calculado para los intervalos de

Figura 1. Consumo específico de oxígeno (CEO) en relación al peso de juveniles Litopenaeus vannamei en tres temperaturas $\left(20,25\right.$ y $\left.30^{\circ} \mathrm{C}\right)$, englobando en cada caso los datos de cuatro salinidades (15, 25, 35 y 45 ups). Las líneas representan la función de regresión / Specific oxygen consumption (CEO) in relation to the weight of Litopenaeus vannamei juveniles at three temperatures $\left(20,25\right.$ and $\left.30^{\circ} \mathrm{C}\right)$, in each case, data of four salinities $(15,25,35$ and $45 \mathrm{psu})$ was utilized. The lines represent the regression function

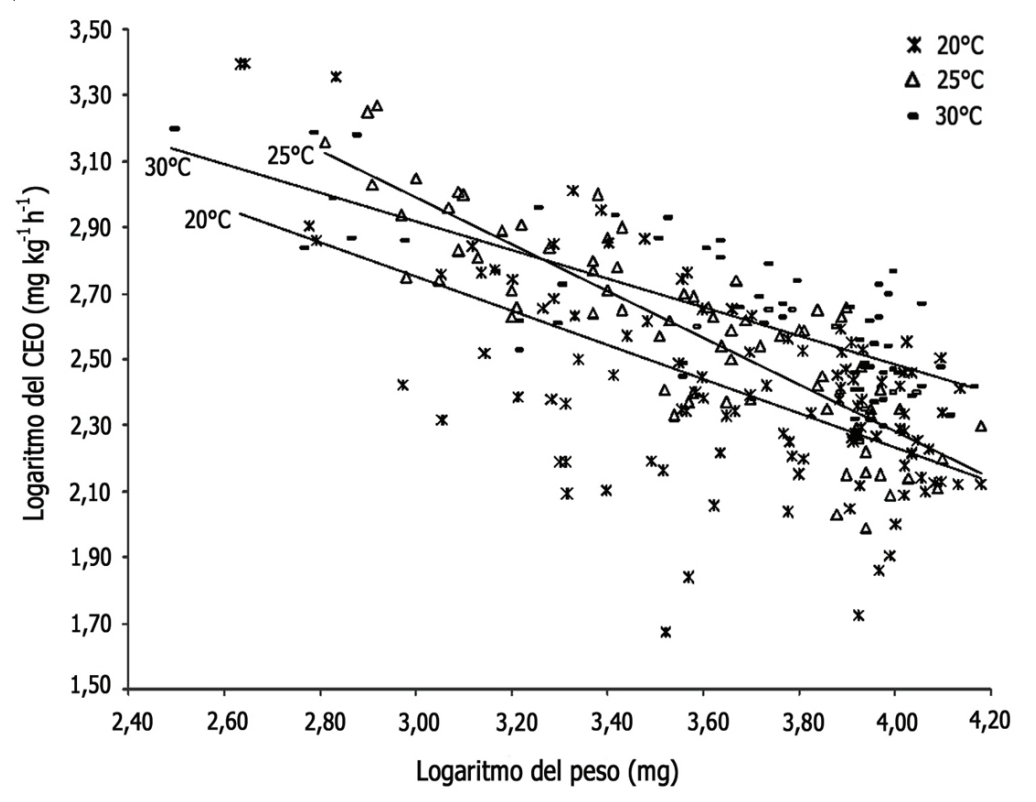

Tabla 2. Modelos de regresión obtenidos para la relación del consumo específico de oxígeno (CEO) y el peso (W) de juveniles Litopenaeus vannamei para tres temperaturas $\left(20,25\right.$ y $\left.30^{\circ} \mathrm{C}\right)$, englobando en cada caso cuatro salinidades $(15,25,35$ y 45 ups) / Regression models obtained for the relationship of specific oxygen consumption (CEO) and weight (W) of Litopenaeus vannamei juveniles combining three temperatures (20, 25 and $\left.30^{\circ} \mathrm{C}\right)$, in each case the data of the four salinities was utilized $(15,25,35$ and $45 \mathrm{psu})$

\begin{tabular}{ccccccc}
\hline Temperatura $\left({ }^{\circ} \mathrm{C}\right)$ & Modelo de regresión & $\mathrm{n}$ & $\mathrm{r}^{2}$ & $\mathrm{~F}$ & $P$ \\
\hline 20 & $\log \mathrm{CEO}=4,29-0,53 \log \mathrm{W}$ & 108 & 0,44 & 90,50 & $<0,0001$ \\
25 & $\log \mathrm{CEO}=5,02-0,69 \log \mathrm{W}$ & 72 & 0,50 & 103,30 & $<0,0001$ \\
30 & $\log \mathrm{CEO}=3,84-0,34 \log \mathrm{W}$ & 68 & 0,28 & 39,20 & $<0,0001$ \\
\hline
\end{tabular}


Tabla 3. Coeficiente termal $\left(Q_{10}\right)$ para el camarón blanco Litopenaeus vananmei expuesto en diferentes combinaciones de temperatura y salinidad / Thermal coefficient $\left(Q_{10}\right)$ for the white shrimp Litopenaeus vananmei exposed to different combinations of temperature and salinity

\begin{tabular}{crrrr}
\hline $\begin{array}{c}\text { Salinidad } \\
\text { (ups) }\end{array}$ & \multicolumn{1}{c}{$\begin{array}{c}\text { Peso } \\
\text { (g) }\end{array}$} & \multicolumn{4}{c}{ Temperatura $\left({ }^{\circ} \mathrm{C}\right)$} \\
\hline 15 & 1 & 0,85 & 0,42 & 0,49 \\
15 & 15 & 0,89 & 0,52 & 0,59 \\
25 & 1 & 1,01 & 0,94 & 0,93 \\
25 & 15 & 1,02 & 0,99 & 0,97 \\
35 & 1 & 2,07 & 1,18 & 0,57 \\
35 & 15 & 1,81 & 1,21 & 0,67 \\
45 & 1 & 1,53 & 0,82 & 0,53 \\
45 & 15 & 1,46 & 0,89 & 0,61 \\
\hline
\end{tabular}

temperatura analizados en el presente estudio, mostró que los camarones pequeños $(\approx 1 \mathrm{~g})$ fueron más sensibles a los cambios de temperatura cuando se expusieron a la salinidad de 35 ups bajo el intervalo $20-25^{\circ} \mathrm{C}$ (Tabla 3 ).

\section{Discusión}

El CEO de los juveniles de camarón Litopenaeus vannamei bajo las combinaciones de temperaturasalinidad aplicadas en este estudio, fue inversamente proporcional al peso de los organismos, lo cual coincide con lo reportado para éste y otros camarones peneidos (Ocampo-Victoria 1994, Martínez-Palacios et al. 1996).

En los camarones expuestos a la salinidad de 35 ups y temperaturas de 20,25 y $30^{\circ} \mathrm{C}$, se observó que en los individuos pequeños $(\approx 1 \mathrm{~g})$ el $\mathrm{CEO}$ aumentó en mayor proporción (1,40 veces) al incrementarse la temperatura de 20 a $25^{\circ} \mathrm{C}$, lo que concuerda con el $\mathrm{Q}_{10}$ calculado en camarones pequeños, que indica que éstos fueron más sensibles a los cambios de temperatura en el intervalo de $20-25^{\circ} \mathrm{C}$ cuando se mantuvieron en un salinidad de 35 ups, mientras que el resto de los organismos tendieron a tolerar mejor las variaciones de temperatura, similar a lo reportado por Bett \& Vinatea (2009).

Por otro lado, los organismos de mayor peso ( 5 y $10 \mathrm{~g})$ proporcionalmente presentaron un menor incremento del CEO (1,19 veces) al pasar de 25 a $30^{\circ} \mathrm{C}$, lo que indica que el efecto de la temperatura sobre el CEO de los organismos dependió de su peso corporal, de tal manera que en organismos pequeños $(1 \mathrm{~g})$ el efecto fue mayor en bajas temperaturas $\left(20 \mathrm{a} 25^{\circ} \mathrm{C}\right)$, a la inversa de lo que sucede en organismos de 5 y $10 \mathrm{~g}$ (mayor efecto de 25 a $30^{\circ} \mathrm{C}$; Tabla 4). Éste efecto no se observó claramente en el trabajo de Martínez-Palacios et al. (1996), ya que el patrón de aumento del CEO tendió a ser semejante tanto en los organismos de 1 como en los de 5 y $10 \mathrm{~g}$ (Tabla 4). Una de las posibles explicaciones que pudieron contribuir a obtener diferencias entre estos trabajos es el origen de los organismos y sus posibles diferencias genéticas, ya que se ha reportado que las tasas respiratorias es una de las características más variables en las especies (Verberg 1983, Randall et al. 1997, Rosas et al. 1999, Salvato et al. 2001, Lemos et al. 2001).

$\mathrm{Al}$ analizar el efecto combinado de temperatura y peso corporal sobre el CEO de los camarones bajo estudio, se observó que un incremento en temperatura generalmente causó modificaciones en el valor de las pendientes de los modelos de regresión simple del CEO. Aunque, el análisis estadístico no mostró diferencias estadísticas significativas entre las pendientes, los valores de estas tendieron a coincidir con lo que se ha observado tanto en esta especie como en otros crustáceos (Tabla 5) y, confirma lo mencionado por Hart (1980), quien reportó que al aumentar la temperatura y el peso corporal generalmente se obtiene una reducción en el valor de las pendientes de los modelos de regresión del CEO. Sin embargo, en otros estudios se ha reportado que cuando varía la temperatura, salinidad o peso de los organismos, la reducción de la pendiente no siempre ocurre (Stephenson \& Knight 1980, Scelzo \& Zuñiga 1987, Gasca-Leyva et al. 1991, MartinezPalacios et al. 1996). Por ejemplo, en Macrobrachium rosenbergi (Stephenson \& Knight 1980) y M. acanthurus (Gasca-Leyva et al. 1991) cuando los organismos se expusieron en agua dulce ( 0 ups), un incremento de temperatura repercutió en una disminución de la pendiente, pero al aumentar la salinidad de 0, 14 a 20 ups, la pendiente ya no mostró tal comportamiento. También, en el presente estudio, se observó que en algunos casos al aumentar la salinidad dentro de cada una de las temperaturas se presentó una tendencia de aumento en el valor de las pendientes (Tabla 5), parecida a lo que se ha reportado con otros crustáceos (Stephenson \& Knight 1980, Gasca-Leyva et al. 1991).

Se ha reportado que tanto en Litopenaeus vannamei como en otros peneidos, al someterlos a salinidades diferentes del punto isosmótico, su gasto energético se incrementa debido a los procesos de osmorregulación, lo que puede reflejarse en un mayor CEO (Valdez et al. 2008, Re et al. 2009). Sin embargo, en el presente estudio, los resultados obtenidos no mostraron un efecto significativo 
Tabla 4. Valores del consumo específico de oxígeno (CEO; $\mathrm{mg} \mathrm{O}_{2}$ $\mathbf{k g}^{-1} \mathbf{h}^{-1}$ ) del presente trabajo y lo reportado por Martínez-Palacios et al. (1996) en Litopenaeus vannamei de 1, 5 y $10 \mathrm{~g}$ en 20,25 y $30^{\circ} \mathrm{C}$ y a salinidad de 35 ups / Values of specific oxygen consumption (CEO; $\mathrm{mg} \mathrm{O}_{2} \mathrm{~kg}^{-1} \mathrm{~h}^{-1}$ ) found in this study and those reported by MartínezPalacios et al. (1996) for Litopenaeus vannamei juveniles of 1, 5 and 10 g at 20,25 and $30^{\circ} \mathrm{C}$ and a salinity of 35 psu

\begin{tabular}{rrrrc}
\hline${ }^{\circ} \mathrm{C}$ & $1 \mathrm{~g}$ & $5 \mathrm{~g}$ & $10 \mathrm{~g}$ & Autor \\
\hline 20 & 1023,29 & 353,72 & 223,87 & Martínez-Palacios et al. (1996) \\
25 & 616,60 & 386,63 & 316,23 & “ \\
30 & 1318,26 & 649,29 & 478,63 & “ \\
20 & 407,38 & 220,99 & 169,82 & El presente estudio \\
25 & 977,24 & 269,65 & 154,88 & “ \\
30 & 912,01 & 502,77 & 389,05 & " \\
\hline
\end{tabular}

Tabla 5. Pendientes de los modelos de regresión del consumo específico de oxígeno (CEO) reportado para Macrobrachium acanthurus y Litopenaeus vannamei en diferentes condiciones de temperatura y salinidad / Slopes of the regression models specific oxygen consumption (CEO) from Macrobrachium acanthurus and Litopenaeus vannamei in different conditions of temperature and salinity

\begin{tabular}{|c|c|c|c|c|c|}
\hline Especie & Peso (g) & $\begin{array}{l}\text { Pendiente de } \\
\text { la regresión }\end{array}$ & $\begin{array}{c}\text { Temperatura } \\
\left({ }^{\circ} \mathrm{C}\right)\end{array}$ & $\begin{array}{l}\text { Salinidad } \\
\text { (ups) }\end{array}$ & Autor \\
\hline \multirow[t]{8}{*}{ Macrobrachium acanthurus } & $0,33-34,00$ & $-0,83$ & 20 & 0 & Gasca-Leyva et al. (1991) \\
\hline & & $-0,69$ & 25 & 0 & \\
\hline & & $-0,68$ & 30 & 0 & \\
\hline & & $-0,53$ & 35 & 0 & \\
\hline & & $-0,70$ & 20 & 20 & \\
\hline & & $-0,67$ & 25 & 20 & \\
\hline & & $-0,55$ & 30 & 20 & \\
\hline & & $-0,75$ & 35 & 20 & \\
\hline \multirow[t]{3}{*}{ Litopenaeus vannamei } & $2,00-24,00$ & $-0,66$ & 20 & 35 & Martínez-Palacios et al. (1996) \\
\hline & $0,80-24,00$ & $-0,30$ & 25 & 35 & \\
\hline & $1,00-50,00$ & $-0,44$ & 30 & 35 & \\
\hline \multirow[t]{12}{*}{ Litopenaeus vannamei } & $1,58-9,77$ & $-0,72$ & 20 & 15 & El presente estudio \\
\hline & $1,41-11,75$ & $-0,56$ & 20 & 25 & \\
\hline & $0,60-15,14$ & $-0,38$ & 20 & 35 & \\
\hline & $0,43-12,30$ & $-0,59$ & 20 & 45 & \\
\hline & $0,81-10,23$ & $-0,58$ & 25 & 15 & \\
\hline & $1,23-14,43$ & $-0,55$ & 25 & 25 & \\
\hline & $0,65-10,72$ & $-0,80$ & 25 & 35 & \\
\hline & $1,26-15,85$ & $-0,83$ & 25 & 45 & \\
\hline & $0,60-11,22$ & $-0,19$ & 30 & 15 & \\
\hline & $1,45-10,72$ & $-0,46$ & 30 & 25 & \\
\hline & $0,66-14,45$ & $-0,37$ & 30 & 35 & \\
\hline & $0,31-10,96$ & $-0,41$ & 30 & 45 & \\
\hline
\end{tabular}


de la salinidad sobre el CEO, tal como ha sido reportado para éste y otros peneidos (Liao \& Murai 1986, Villarreal \& Rivera 1992, Villarreal et al. 1992, Bett \& Vinatea 2009). En contraste, existen reportes que indican que en crustáceos se puede presentar una disminución en el CEO en salinidad supra y/o subnormal (Verberg 1983). Tales diferencias entre la información pueden ser debido a que la valoración del CEO se ha realizado en organismos con diferente origen (silvestre, laboratorio o de cultivo), condición de salud, etapa del ciclo de muda, entre otras variables, lo cual repercute sobre la condición fisiológica que se refleja en el CEO.

En conclusión, el CEO disminuyó con el aumentó del peso corporal de los organismos en todos los tratamientos, sin presentar un patrón general definido de aumento del CEO conforme se incrementó la salinidad en cada una de las temperatura ensayadas. Aunque no se presentaron diferencias estadísticas significativas en las pendientes de los modelos de regresión calculados para expresar la dependencia del CEO en relación al peso de los organismos, los resultados sugieren que: a $20^{\circ} \mathrm{C}$ los camarones presentaron mayor gasto energético en la menor salinidad ( $15 \mathrm{ups}$ ), a $25^{\circ} \mathrm{C}$ ocurrió el mayor gasto energético en la salinidad más alta (45 ups), mientras que en $30^{\circ} \mathrm{C}$ se observó el menor gasto energético en la menor salinidad (15 ups). Esto indica que la condición isosmótica (i.e., punto de menor gasto energético, Castille \& Lawrence 1981) tiende a modificarse con la temperatura. Además, en este estudio los resultados indican que los camarones pueden encontrar un nivel óptimo de cultivo a alta temperatura $\left(30^{\circ} \mathrm{C}\right)$ y baja salinidad ( 15 ups) debido al gasto energético observado. Además, sería importante a futuro caracterizar el efecto de la temperatura-salinidad sobre el CEO bajo diferentes condiciones de salud, origen de los organismos y sexo, ya que las tasas respiratorias de un mismo grupo de organismos presentaron una amplia variación cuando se sometieron a una misma condición ambiental.

\section{Agradecimientos}

Se agradece al Consejo Estatal de Ciencia y Tecnología de Sinaloa (CECyT 2007-2010) e Instituto Politécnico Nacional (Proyectos SIP-20080290, 20090864 y 20100145) por aportar fondos para culminar la presente investigación. También se agradece al laboratorio productor de larvas Yameto S.A. de C.V., por proveernos camarón.

\section{LITERATURA CITADA}

Bett C \& L Vinatea. 2009. Combined effect of body weight, temperature and salinity on shrimp Litopenaeus vannamei oxygen consumption rate. Brazilian Journal of Oceanography 57: 305-314.

Boyd CE \& BJ Watten. 1989. Aeration systems in aquaculture. Critical Review of Aquatic Science 1: 425-438.

Castille Jr \& AL Lawrence. 1981. The effect of salinity on the osmotic, sodium and chloride concentrations in the hemolymph of euryhaline shrimp of the genus Penaeus. Comparative Biochemistry and Physiology 68: 75-80.

Chen JC \& SH Lai. 1993. Effects of temperature and salinity on oxygen consumption and ammonia-N excretion of juvenile Penaeus japonicus Bate. Journal of Experimental Biology and Ecology 165(2): 161-170.

Esparza-Leal HM, JT Ponce-Palafox, EAAragón-Noriega, JL Arredondo-Figueroa, M García-Ulloa Gómez \& W Valenzuela-Quiñónez. 2010. Growth and performance of the whiteleg shrimp Penaeus vannamei (Boone) cultured in low-salinity water with different stocking densities and acclimation times. Aquaculture Research 41: 878-883.

Gasca-Leyva JFE, CA Martínez-Palacios \& LG Ross. 1991. The respiratory requirements of Macrobrachium acanthurus (Weigman) at different temperature and salinity. Aquaculture 93: 191-197.

Grasshoff K. 1976. Methods of seawater analysis, 317 pp. Verlag Chemie, Weinheim, New York.

Hart RC. 1980. Oxygen consumption in Caridina nilotica (Decapoda, Atydae) in relation to temperature and size. Freshwater Biology 10: 215-222.

Hemerick G. 1973. Mass culture. En: Stein JR (ed). Handbook of phycological methods, pp. 255-273. Cambridge University Press, New York.

Kurmaly K, AB Yule \& DA Jones. 1989. Effects of body size and temperature on the metabolic rate of Penaeus monodon. Marine Biology 103: 25-30.

Kutty MN, G Murugopoopathy \& TS Krisnan. 1971. Influence of salinity and temperature on the oxygen consumption in young juveniles of the Indian prawn Penaeus indicus. Marine Biology 11: 125-131.

Lemos D, VN Phan \& G Alvarez. 2001. Growth, oxygen consumption, ammonia-N excretion, biochemical composition and energy content of Farfantepenaeus paulensis Pérez-Farfante (Crustacea, Decapoda, Penaeidae) early postlarvae in different salinities. Journal of Experimental Marine Biology and Ecology 261: 55-74.

Li E, L Chen, C Zeng, X Chen, N Yu, Q Lai \& JG Qin. 2007. Growth, body composition, respiration and ambient ammonia nitrogen tolerance of the juvenile white shrimp, Litopenaeus vannamei, at different salinities. Aquaculture 265: 385-390.

Liao YC \& T Murai. 1986. Effects of dissolved oxygen, temperature and salinity on the oxygen consumption of the grass shrimp Penaeus monodon: En: Maclean JL, LB Dizon \& LV Hosillos (eds). Forum Asian Fisheries Society, pp. 641-646. Asian Fisheries Society, Manila. 
Martínez-Palacios CA, LG Ross \& L Jimenez-Valenzuela. 1996. The effects of temperature and body weight on the oxygen consumption of Penaeus vannamei Boone, 1931. Journal of the Aquaculture in the Tropics 11: 59-65.

Ocampo-Victoria L. 1994. Evaluación de la temperatura y el peso en el consumo de oxígeno del camarón café Penaeus californiensis (Holmes, 1900) (Decapoda: Penaeidaea). Tesis de Maestría, Centro Interdisciplinario de Ciencias Marinas-IPN. La Paz, Baja California, 135 pp.

Randall D, W Burggen \& H French. 1997. Fisiología animal: Mecanismos e adaptacoes, 729 pp. Rio de Janeiro: Guanabara Koogan.

Re AD \& F Díaz. 2011. Effect of different oxygen concentrations on physiological energetics of blue shrimp, Litopenaeus stylirostris (Stimpson). The Open Zoology Journal 4: 1-8.

Re AD, F Díaz, E Sierra \& S Gómez-Jiménez. 2004. Consumo de oxígeno, excreción de amonio y capacidad osmorreguladora de Litopenaeus stylirostris (stimpson) expuesto a diferentes combinaciones de temperatura y salinidad. Ciencias Marinas 30: 443-453.

Re AD, F Díaz, G Valdez, M Flores \& M López. 2009. Physiological energetics of blue shrimp Penaeus stylirostris (Stimpson) juveniles acclimated to different salinities. The Open Zoology Journal 2: 102-108.

Robaina OG. 1983. Efectos de la salinidad y la temperatura en la sobrevivencia del camarón Penaeus brasiliensis Latreille (Crustacea, Decapoda, Penaeidea). Revista Latinoamericana de Acuicultura 17: 25-37.

Robertson L, W Bray, J Leung-Trujillo \& A Lawrence. 1987. Practical molt staging of Penaeus setiferus and Penaeus stylirostris. Journal of the World Aquaculture Society 18: 180-185.

Rosas C, E Martínez, G Gaxiola, R Brito, A Sánchez \& LA Soto. 1999. The effect of dissolved oxygen and salinity on oxygen consumption, ammonia excretion and osmotic pressure of Penaeus setiferus (Linnaeus) juveniles. Journal of Experimental Marine Biology and Ecology 234: 41-57.

Rosas C, E Martínez, M Aguilar, A Sánchez, R Días-Iglesia, E Brito \& ELA Martínez-Soto. 1997. Critical dissolved level to Penaeus shcmitti postlarvae $\left(\mathrm{PL}_{10-18}\right)$ exposed to salinity changes. Aquaculture 152: 259-272.

Ross B \& LG Ross. 1983. The respiratory performance of Oreochromis niloticus under adverse environmental conditions. En: Proceeding of the First International Symposium on Tilapia in Aquaculture, pp. 134-143. Tel Aviv University Press, Tel Aviv.

Salvato B, V Cuomo, P Di Muro \& M Beltrami. 2001. Effect of environmental parameters on the oxygen consumption of four marine invertebrates: a comparative factorial study. Marine Biology 138: 659-668.
Scelzo MA \& O Zuñiga. 1987. Consumo de oxígeno en el camarón Penaeus brasiliensis Latreille (Decapoda: Penaeidae) en relación a salinidad y temperatura. En: Memorias de la Sociedad de Ciencias Naturales de la Universidad La Salle, pp. 127-128. Universidad La Salle, México.

Schmidt-Nielsen K. 1997. Animal physiology: adaptation and environment, 607 pp. Cambrigde University Press, Cambridge.

Sokal RR \& FJ Rohlf. 1981. Biometry. The principles and practice of statistic in biological research, $859 \mathrm{pp}$. Freeman WH \& Company, New York.

Spanopoulos-Hernández M, CA Martínez-Palacios, RC Venegas-Pérez, C Rosas \& LG Ross. 2005. The combined effect of salinity and temperature on the oxygen consumption of juvenile shrimps Litopenaeus stylirostris (Stimpson, 1874). Aquaculture 244: 341-348.

Steel RGD \& JH Torrie. 1980. Principles and procedures of statistics, a biometrical approach, 2nd. edition, $428 \mathrm{pp}$. McGraw-Hill Book Company, New York.

Stephenson MJ \& AW Knight. 1980. The effect of temperature and salinity on oxygen consumption of post-larvae of Macrobrachium rosenbergi (de Man) (Crustacea: Palaemonidae). Comparative Biochemistry and Physiology 67: 699-703.

Summerfelt ST, BJ Vinci \& RH Piedrahita. 2000. Oxygenation and carbon dioxide control in water reuse systems. Aquacultural Engineering 22: 87-108.

Valdez G, F Diaz, AD Re \& E Sierra. 2008. Efecto de la salinidad sobre la fisiología energética del camarón blanco Litopenaeus vannamei (Boone). Hidrobiológica 18: 105115.

Vernberg FJ. 1983. Respiratory adaptations. The biology of Crustacea, environmental adaptations, 44 pp. Academic Press, New York.

Villarreal H \& A Rivera. 1992. Effects of temperature and salinity on the oxygen consumption of laboratory produced Penaeus vannamei postlarvae. En: Aquaculture'92. Growing Toward the 21st Century, pp. 224-225. World Aquaculture Society, Orlando.

Villarreal H, J Naranjo \& P Hinojosa. 1992. Effects of temperature and salinity on the oxygen consumption of laboratory produced Penaeus californiensis postlarvae. En: Aquaculture'92. Growing Toward the 21st Century, 225 p. World Aquaculture Society, Orlando.

Yan B, X Wang \& M Cao. 2007. Effect of salinity and temperature on survival, growth, and energy budget of juvenile Litopenaeus vannamei. Journal of Shellfish Research 26: 141-146.

Zar JH. 1984. Biostatistical analysis, 718 pp. Prentice-Hall, Englewood Cliffs.

Recibido el 2 de abril de 2011 y aceptado el 30 de agosto de 2011 